\title{
Total Serum Protein as an Independent Predictor of Heart Failure with Preserved Ejection Fraction in Obese Pediatric Population
}

Poruban $\mathbf{T}^{1 *}$, Banovcinova $\mathrm{A}^{2}$, Vachalcova $\mathbf{M}^{1}$, Sieradzka AK ${ }^{1}$, J akubova $M^{1}$, Schusterova $I^{1,2}$ and Barkai LL ${ }^{2}$

${ }^{1}$ East Slovak Institute of Cardiovascular Diseases, Kosice, Slovakia

${ }^{2}$ Department of Pediatrics and Adolescent Medicine, Faculty of Medicine, Pavol J ozef Safarik University, Kosice, Slovakia

*Corresponding author: Poruban T, East Slovak Institute of Cardiovascular Diseases, Ondavska 8, Kosice, Slovakia

Received: September 01, 2021; Accepted: October 28, 2021; Published: November 04, 2021

\begin{abstract}
Objectives: We aimed to clarify the prognostic role of Total Serum Protein (TSP) in obese children with HFpEF and its using as an effective and noninvasive approach for screening of target population.

Methods: In total, 587 patients who enrolled in our unique program aimed for children's obesity treatment were referred. Among these patients, we identified and retrospectively studied 64 patients who met our criteria and compared them with 24 lean healthy subjects. Baseline examination, routine blood testing and transthoracic echocardiography were obtained.

Results: We revealed that obese patients had higher TSP levels compared to them with normal weight. They also had worse echocardiographic results including a lower Left Ventricular Ejection Fraction (LVEF) and E/A ratio. Positive correlations between TSP and Pulmonary Artery Systolic Pressure (PASP), Left Atrial Volume Index (LAVI), and Interventricular Septal Systolic/Diastolic Dimension (IVSs, IVSd) and negative TSP correlations with LVEF and E/A ratio were found, too. Compared to the commonly used Albumin-to-Globulin Ratio (AGR), the TSP was a better metabolic predictor. There were more significant correlations in obese subgroup with HFpEF than to those without HFpEF.
\end{abstract}

Conclusions: We first indicated that higher TSP levels are positively associated with obesity and HFpEF in children and could be used as a more easily available biomarker which provides a more-accurate HFpEF risk evaluation of obese pediatric population group than other objective indices, possibly allowing early implementation of appropriate intervention in daily practice and leads to better outcomes and early prevention in patients with higher HF risk.

Keywords: Heart failure; Preserved ejection fraction; Obesity; Total serum protein

\section{Introduction}

Reduced Ejection Fraction (EF) has traditionally been used to represent Heart Failure (HF) syndromes, but it is now widely acknowledged that nearly Half of HF patients have Preserved EF (HFpEF). The diagnosis of HFpEF requires the following conditions to be satisfied: (1) signs or symptoms of HF; (2) normal or mildly abnormal systolic Left Ventricular (LV) function; (3) evidence of diastolic LV dysfunction [1]. Epidemiological studies have suggested a high prevalence of HFpEF in adults $(1.1-5.5 \%)$, incidence has increased over the past decades [2,3]. Numerous studies have enhanced understanding of HFpEF [4-10]. However, all of them have been conducted in adults, and there are very limited informations regarding HFpEF in children [11]

Childhood obesity has reached epidemic proportions worldwide [12]. It has long been described as a major comorbidity in HFpEF patients [13-15]. Obesity has been proposed as a major driver of systemic inflammation, ultimately predisposing to myocyte remodeling and the development of HFpEF [16-19]. Obesity and HFpEF is substantiated by prior community-based studies, demonstrating an association of obesity with future HFpEF [2022]. Obesity and related cardio-metabolic traits are also more strongly associated with the risk of future HFpEF rather than HFrEF, suggesting that obesity-associated HFpEF represents a distinct clinical phenotype within the broad spectrum of HFpEF [23,24].

However, in epidemiological studies mild to moderate overweight or obesity status was reported to have a protective effect in patients with HF $[25,26]$. This phenomenon was termed "the obesity paradox" and initially observed in small population studies [27,28] and confirmed in large observational studies in both HFrEF and HFpEF patients [29-31]. But other studies have not shown that the obesity paradox exists in HFpEF [32-34], and thus, the causal link between this scientific observation and its clinical implications are limited and remain hotly debated. Several hypotheses are proposed to explain the presence or absence of the obesity paradox $[35,36]$, and have been extensively reviewed [37-39].

Because of the potential cardiovascular consequences associated with obesity, it is vital to identify children at risk of HFpEFidentification of promising prognostic factors could improve their 
long-term survival. Numerous prognostic markers associated with HF have been identified, but their clinical applicability is limited and precise risk stratification remains challenging [40-42]. There is a lack of consensus on how we define HFpEF. This lack of uniformity in disease definition stems in part from an incomplete understanding of disease pathobiology, phenotypic heterogeneity, and natural history. Although most criteria rely on the presence of clinical symptoms and preserved ejection fraction, there is substantial variation regarding the use of biomarkers, abnormal cardiac structure and function ascertained by echocardiography, and previous hospitalizations to define HFpEF [43-45]. Unlike other diseases within cardiovascular medicine (atrial fibrillation, hypertension, etc.) where definitions are centered around a specific diagnostic test, HFPEF is a clinical syndrome for which we rely on a constellation of symptoms, signs, and other manifestations. Thus, simple but effective prognostic biomarker models are needed to improve the management of the HF epidemic.

In recent studies, the correlation between Serum Albumin (sALB) and Globulins (sGLB), commonly used in clinical practice as Albumin-Globulin Ratio (AGR), has been confirmed to be associated with impaired survival in patients with HF [46]. However, the TSP, including not only sALB and sGLB, but also other inflammatory proteins, as a cumulative and effective prognostic biomarker in early diagnosis of HF has not been studied previously [47]. What is more, TSP measurement is often not included in the routine battery of blood tests of cardiac patients, presumably because interpretations may be uncertain in a clinical setting. There is an ongoing debate whether TSP can be used as a causal risk factor, or merely a nonspecific marker of disease.

The hypothesis underlying the current study was that HFpEF status can be accessed via total protein level in serum that assess multiple pathways of disease as a low-risk, non-invasive approach for screening of obese children.

\section{Methods}

\section{Patients}

All medical records of patients were referred between August 20, 2017 and December 15, 2019 to the School of Obesity-a unique interdisciplinary outpatient program aimed for children's obesity treatment supervised by the Department of Metabolic Disease of the Pediatric Clinic at the Children's Faculty Hospital, Kosice, since 2017 as the only one of its type in Slovakia. The programme has been performing according to the Declaration of Helsinki, and the hospital ethics review board approved the protocol. Only patients whose parents signed the approved voluntary informed consent document for this study have been including.

The data were analyzed retrospectively to identify pediatric patients ( $<18$ years old) with HFpEF. HFpEF was defined as (1) HF signs or symptoms with LVEF $>50 \%$ and (2) objective evidence of diastolic dysfunction obtained by echocardiography [48]. Clinical signs and symptoms of HF were based on a modification of the previously described criteria in adults: history of acute pulmonary edema, or the presence of at least 2 of the following clinical features with no other identifiable cause and improvement following diuresis: dyspnea, bilateral edema of the lower extremities, or hepatomegaly.
64 subjects who were enrolled in our School of Obesity program were included with following inclusion criteria: under the age of 18 years, diagnosis of overweight or obesity, absence of comorbidities and were compared with 24 lean healthy subjects. Exclusion criteria were as follows: (1) unavailable data of baseline TSP levels, (2) previously diagnosed at least one of any following diseases: significant hematological disorders, thyroid dysfunction, liver or renal insufficiency, infectious or systemic inflammatory diseases and malignant tumors, (3) history of surgical correction of cardiovascular lesions, (4) hypertrophic/restrictive cardiomyopathies, (5) chromosomal abnormalities or (6) interrupted cooperation during follow-up. Lean controls were healthy children matched for age and sex, in whom lipid and glucose metabolism disorders or obesity were not presented.

All patients had to be followed up every 3 months for the 12 months by the trained nurses or cardiologists who were blinded to the aim of this study. The same analysis was performed in the control group and the results were compared.

\section{Data extraction and baseline examination}

Data regarding patient demographics, echocardiographic examination, and laboratory measurements including TSP levels were extracted from medical records.

Height and weight were measured, and Body Mass Index (BMI) $\left(\mathrm{kg} / \mathrm{m}^{2}\right)$ was calculated as Weight $(\mathrm{kg})$ divided by the square of height $\left(\mathrm{m}^{2}\right)$. BMI percentiles and Waist Circumference (WC) were measured according to World Health Organization's recommendations [49,50]. Overweight was defined as a BMI at or above the $85^{\text {th }}$ percentile and below the $95^{\text {th }}$ percentile for children and teens of the same age and sex. Obesity was defined as a BMI at or above the $95^{\text {th }}$ percentile for children and teens of the same age and sex [51].

Blood Pressure (BP) was measured with a standard oscillometric sphygmomanometer, and stethoscope placed over the brachial artery pulse, proximal and medial $2 \mathrm{~cm}$ above the cubital fossa. The cuff used was appropriate for the size of the child's upper right arm. Systolic and diastolic BP were measured three times after 10 min rest in the supine position, according to the recent recommendations and the average of the three measurements was calculated [52]. Hypertension was defined as $\mathrm{BP} \geq 95^{\text {th }}$ percentile to $<95^{\text {th }}$ percentile $+12 \mathrm{mmHg}$ or $130 / 80$ to $139 / 89 \mathrm{mmHg}$ (for children aged 1-12 years) or as $\mathrm{BP}>130$ 139/80-89 mmHg (for children aged 13 years and older) [53].

\section{Biochemical measurements}

Serum was isolated from blood samples collected after overnight fasting. Venous blood $(5 \mathrm{ml})$ was drawn into a red top vacutainer serum tube and placed upright 30 to 60 minutes until clot formation. The tubes were centrifuged in a swinging bucket rotor $(1.300 \mathrm{~g} \mathrm{x} 20$ $\mathrm{min}$ ) and the serum was pipetted into $1.5 \mathrm{ml}$ vials. Plasma glucose, serum Triglycerides (TAG), Total Cholesterol (TC) and High-Density Lipoprotein (HDL) cholesterol concentrations were measured with standard laboratory techniques on colorimetric enzymatic assay systems (Siemens ADVIA 1800, Siemens Healthineers, Erlangen, Germany). Low Density Lipoprotein (LDL) cholesterol was calculated according to Fridewald's formula [54]. Fasting serum insulin was measured by a sandwich ECLA method (Roche MODULAR E170, Hoffmann-La Roche AG, Basel, Switzerland). 
Aspartate aminotransferase (AST) and alanine aminotransferase (ALT) were measured by photometric kinetic methods (Siemens ADVIA 1800). The levels of albumin (sAlb), globulin (sGlb) and TSP were quantitatively measured by the method based on the biuret reaction, in which an alkaline copper solution reacts with peptide linkages to form a complex that absorbs light at wavelength $540 \mathrm{~nm}$. The sensitivity of reaction was increased in accordance with Lowry method - by the addition of phosphotungstomolybdic acid (FolinCiocalteu / phenol reagent).

DM was defined as a fasting plasma glucose $\geq 126 \mathrm{mg} /$ dl $(7.0 \mathrm{mmol} / \mathrm{l})$ in multiple determinations [55]. Dyslipidemia was considered to be present in children if they had fasting total cholesterol $\geq 200 \mathrm{mg} / \mathrm{dl}$ ( $5.0 \mathrm{mmol} / \mathrm{l})$ or triglyceride $\geq 150 \mathrm{mg} / \mathrm{dl}$ (3.75 $\mathrm{mmol} / \mathrm{l}$ ) [56]. Presence of Metabolic Syndrome (MS) was determined according to IDF 2007 criteria [57].

\section{Echocardiography}

Two-dimensional transthoracic echocardiographic examinations were obtained in all subjects in calm state and in the left lateral decubitus position using a Siemens Acuson SC 2000 Prime ultrasound system, with a $2.5 \mathrm{MHz}$ transducer (Siemens Healthineers, Erlangen, Germany), with a frame rate $\geq 50$ frame/sec. All measurements were performed according to the recommendations of the American Society of Echocardiography [58]. All images were digitally stored with at least three cardiac cycles for offline analysis. The conventional recorded parameters included the Left Ventricular Ejection Fraction (LVEF), E/A ratio, Left Atrial Volume Index (LAVI), Pulmonary Artery Systolic Pressure (PASP), and Interventricular Septal Diastolic and Systolic Dimensions (IVSd, IVSs). LVEF was assessed by the biplane Simpson's method, E/A ratio was assessed by color M-mode Doppler. PASP was calculated as $4^{\star}$ (peak TR velocity) ${ }^{2}$. As a cutoff value to identify HFpEF was considered $\mathrm{EF} \geq 50 \%$ by the end of follow-up. Cut-off values for all recorded parameters were considered according to recent studies [59]. The imaging procedures were conducted by the same professional echocardiographer, masked to the cohort data.

\section{Statistical analysis}

Data were processed using methods of descriptive and inductive statistics, depending on the type and number of monitored variables. For the purpose of inductive statistics, we assumed that our data represent a random sample of the relevant population. The first step was a one-dimensional analysis - the tabulation of all monitored variables using frequency tables. The second step was a twodimensional analysis - the assessment of pairs of monitored variables. To compare numerical and categorical variables (e.g. obesity level), analysis of variance was used to determine the statistical significance of differences, if the distribution of variables was normal. The last step was a multidimensional analysis-a multiple linear regression analysis, where the relationship between several numerical variables was examined simultaneously. Therefore, EF is presented as a dependent (outcome) variable, the baseline and biochemical parameters including TSP as independent (explanatory) variables. Statistical analysis was conducted using Prism 8 (GraphPad Software Inc, San Diego, CA). All of the statistical tests were considered statistically significant if $\mathrm{p}<0.05$. Data were summarized as means \pm SD.

\section{Results}

In total, 587 patients were referred to our School of Obesity programme from August 2017 through December 2019. Among these patients, we identified 64 patients who met inclusion/exclusion criteria, including 4 with HFpEF (6.25\%). The demographic data of these patients are summarized in (Table 1). Compared with the patients without obesity, the ones who were obese had higher levels of TSP $(76.5 \pm 4.5$ vs. $71.5 \pm 3.5, \mathrm{p}<0.05)$. We also found that body weight, BMI, BMI percentile, WC and systolic and diastolic BP were significantly higher in obese subjects compared to lean controls. Obese (and overweight) subjects had higher levels of ALT, TAG, total and LDL cholesterol compared to control group, while HDL cholesterol was lower.

We found significant differences in the distribution of TSP compared to them with normal weight, as well as sAlb and AGR (Table 2). In this pilot study, simple linear regression analysis showed a positive correlation of TSP with TC. Positive correlations were also found between TSP and sAlb, AGR, respectively. Multiple regression analysis revealed that after adjusting for BW, BMI, BMI percentile, WC and systolic and diastolic BP, sAlb $(\mathrm{r}=0.36, \mathrm{p}<0.05)$ and AGR $(\mathrm{r}=0.57, \mathrm{p}<0.05)$ were the only two markers correlating with TSP (Table 3).

Table 1: Baseline characteristics.

\begin{tabular}{|c|c|c|}
\hline Parameter & $\begin{array}{c}\text { Obese/Overweight (Ow) } \\
\text { Group } \\
(\mathrm{n}=64)\end{array}$ & $\begin{array}{c}\text { Control Group (n } \\
=24)\end{array}$ \\
\hline HFpEF confirmed & 4 & 1 \\
\hline Age (years) & $13.2 \pm 5.3^{* *}$ & $12.8 \pm 6.2$ \\
\hline Body weight (kg) & $76 \pm 15.9^{* *}$ & $59.8 \pm 13.4$ \\
\hline Height (cm) & $157.1 \pm 15.6^{* *}$ & $150.4 \pm 12.6$ \\
\hline BMI $\left(\mathrm{kg} / \mathrm{m}^{2}\right)$ & $29.4 \pm 4.4^{* *}$ & $25.8 \pm 4.1$ \\
\hline BMI percentile & $95.1 \pm 3.5^{* *}$ & $37.1 \pm 2.9$ \\
\hline BMI Z-score & $-0.41 \pm 1.03^{* *}$ & $2.06 \pm 0.54$ \\
\hline $\begin{array}{l}\text { Waist circumference } \\
(\mathrm{cm})\end{array}$ & $93.6 \pm 12.9^{* *}$ & $70.5 \pm 9.6$ \\
\hline Systolic BP (mmHg) & $126.6 \pm 16.3^{*}$ & $120.8 \pm 16.6$ \\
\hline Diastolic BP (mmHg) & $73.5 \pm 10.4^{* *}$ & $69.9 \pm 6.4$ \\
\hline $\begin{array}{l}\text { Fasting glucose } \\
(\mathrm{mmol} / \mathrm{l})\end{array}$ & $4.7 \pm 0.4$ & $4.6 \pm 1.4$ \\
\hline Fasting insulin (IUI/I) & $18.2 \pm 6.9^{*}$ & $7.5 \pm 4.6$ \\
\hline $\begin{array}{l}\text { Total cholesterol } \\
(\mathrm{mmol} / \mathrm{l})\end{array}$ & $4.5 \pm 1.0^{*}$ & $4.4 \pm 0.6$ \\
\hline Triglyceride (mmol/l) & $1.29 \pm 0.8^{* *}$ & $0.9 \pm 0.4$ \\
\hline $\begin{array}{l}\text { HDL cholesterol } \\
(\mathrm{mmol} / \mathrm{l})\end{array}$ & $1 \pm 0.2^{*}$ & $1.5 \pm 0.2$ \\
\hline $\begin{array}{l}\text { LDL cholesterol } \\
(\mathrm{mmol} / \mathrm{l})\end{array}$ & $3.3 \pm 1.2^{*}$ & $2.7 \pm 0.6$ \\
\hline AST (U/l) & $31 \pm 13$ & $25 \pm 11$ \\
\hline ALT (U/l) & $40 \pm 29^{*}$ & $19 \pm 7$ \\
\hline sAlb $(g / l)$ & $45.4 \pm 4.2^{*}$ & $40.2 \pm 3.7$ \\
\hline AGR & $1.8 \pm 0.4^{* * * *}$ & $1.5 \pm 0.4$ \\
\hline TSP $(g / l)$ & $76.5 \pm 4.5^{*}$ & $71.5 \pm 3.5$ \\
\hline
\end{tabular}

Data are mean as \pm SD. ${ }^{*} \mathrm{p}<0.05 ;{ }^{* *} \mathrm{p}<0.01 ;{ }^{* * *} \mathrm{p}<0.001 ;{ }^{* * * *} \mathrm{p}<0.0001$.

AGR: Albumin-To-Globulin Ratio; ALT: Alanine Aminotransferase; AST: Aspartate Aminotransferase; BMI: Body Mass Index; BP: Blood Pressure; HDL: High- Density Lipoprotein; LDL: Low-Density Lipoprotein; sAlb: Serum Albumin; TSP: Total Serum Protein. 
Table 2: Correlation between total serum protein (TSP) and baseline characteristics.

\begin{tabular}{|c|c|c|}
\hline \multirow{2}{*}{ Parameter } & $\begin{array}{l}\text { TSP in Obese/Ow } \\
\text { Group }(\mathrm{N}=64)\end{array}$ & $\begin{array}{l}\text { TSP in Control } \\
\text { Group }(\mathrm{N}=24)\end{array}$ \\
\hline & $\mathbf{r}$ & $\mathbf{r}$ \\
\hline Body weight (kg) & 0.08 & 0.27 \\
\hline Height (cm) & 0.02 & 0.29 \\
\hline BMI $\left(\mathrm{kg} / \mathrm{m}^{2}\right)$ & 0.16 & 0.18 \\
\hline BMI percentile & 0.24 & 0.27 \\
\hline Waist circumference $(\mathrm{cm})$ & 0.14 & 0.12 \\
\hline Systolic BP (mmHg) & -0.01 & 0.04 \\
\hline Diastolic BP (mmHg) & -0.22 & -0.19 \\
\hline Fasting glucose $(\mathrm{mmol} / \mathrm{l})$ & -0.23 & -0.01 \\
\hline Fasting insulin (IUI//) & -0.02 & $<0.05$ \\
\hline Total cholesterol $(\mathrm{mmol} / \mathrm{l})$ & $0.47^{*}$ & $0.42^{*}$ \\
\hline Triglyceride (mmol/l) & 0.31 & 0.08 \\
\hline HDL cholesterol (mmol/l) & 0.23 & 0.1 \\
\hline LDL cholesterol (mmol/l) & -0.04 & 0.19 \\
\hline AST (U/l) & 0.16 & 0.07 \\
\hline $\operatorname{ALT}(U / I)$ & 0.03 & $<0.05$ \\
\hline sAlb $(g / l)$ & $0.68^{*}$ & $0.59^{* *}$ \\
\hline AGR & $0.65^{*}$ & $0.63^{* *}$ \\
\hline
\end{tabular}

Data are mean as \pm SD. ${ }^{*} p<0.05 ;{ }^{*} p<0.01$

AGR: Albumin-To-Globulin Ratio; ALT: Alanine Aminotransferase; AST: Aspartate Aminotransferase; BMI: Body Mass Index; BP: Blood Pressure, HDL: High Density Lipoprotein; LDL: Low-Density Lipoprotein; R: Correlation Coefficient sAlb: Serum Albumin; TSP: Total Serum Protein.

Table 3: Multiple regression analysis of some metabolic parameters (pooled groups).

\begin{tabular}{|l|c|c|c|}
\hline \multirow{2}{*}{\multicolumn{1}{|c|}{ Parameter }} & \multicolumn{3}{c|}{ TSP } \\
\cline { 2 - 4 } & $\mathbf{r}$ & tc & ß coefficient \\
\hline Total cholesterol (mmol/l) & 0.65 & 0.47 & 0.91 \\
\hline SAlb $(\mathrm{g} / \mathrm{l})$ & $0.36^{*}$ & 2.85 & -0.08 \\
\hline AGR & $0.57^{\star}$ & 1.12 & -0.11 \\
\hline
\end{tabular}

*p<0.05.

AGR: Albumin-To-Globulin Ratio; r: Correlation Coefficient, sAlb: Serum Albumin tc: Thigh Circumference; TSP: Total Serum Protein.

Table 4 compared echocardiographic characteristics between obese (and overweight) and lean subjects. For this entire cohort, the participants had a worse echocardiographic results including a lower $\operatorname{LVEF}(60.8 \pm 10.6 v s .63 .5 \pm 10.1, \mathrm{p}<0.001)$ and $\mathrm{E} / \mathrm{A}$ ratio $(1.67 \pm 0.43$ vs. $2.05 \pm 0.56, \mathrm{p}<0.05)$ than the lean group.

Simple linear regression analysis showed positive correlations of TSP with PASP, LAVI, IVSs and IVSd. Negative correlations of TSP with LVEF and E/A ratio (Table 5). All findings were confirmed using multiple regression analysis, too (Table 6).

As shown in (Table 7), TSP was a better metabolic predictor than AGR not only in relationship with LVEF, but also with other metabolic and echocardiographic parameters, but AGR showed a similar predictive value as TSP (Table 8). shows more significant correlations in obese subgroup with HFpEF than to those without HFpEF.
Table 4: Echocardiographic characteristics.

\begin{tabular}{|l|c|c|}
\hline \multicolumn{1}{|c|}{ Parameter } & $\begin{array}{c}\text { Obese/Ow Group } \\
(\mathbf{N}=64)\end{array}$ & $\begin{array}{c}\text { Control Group } \\
(\mathbf{N}=\mathbf{2 4})\end{array}$ \\
\hline LVEF $(\%)$ & $60.8 \pm 10.6^{* * *}$ & $63.5 \pm 10.1$ \\
\hline $\begin{array}{l}\text { LV diastolic dimension } \\
(\mathrm{mm})\end{array}$ & $47.5 \pm 3.3^{*}$ & $43.4 \pm 4.4$ \\
\hline Relative wall thickness & $0.36 \pm 0.04^{*}$ & $0.32 \pm 0.03$ \\
\hline LV mass index $\left(\mathrm{g} / \mathrm{m}^{2}\right)$ & $38.5 \pm 9.5^{*}$ & $34.2 \pm 8.9$ \\
\hline E/A ratio & $1.67 \pm 0.43^{*}$ & $2.05 \pm 0.56$ \\
\hline LAVI $\left(\mathrm{ml} / \mathrm{m}^{2}\right)$ & $28.5 \pm 8.1^{* * *}$ & $27.2 \pm 8.3$ \\
\hline PASP $(\mathrm{mmHg})$ & $31.7 \pm 5.2^{*+*}$ & $31.3 \pm 4.9$ \\
\hline IVSs $(\mathrm{mm})$ & $11.6 \pm 6.1^{* * *}$ & $12.5 \pm 7.0$ \\
\hline IVSd $(\mathrm{mm})$ & $11.9 \pm 6.0^{* * *}$ & $11.6 \pm 2.0$ \\
\hline
\end{tabular}

Data are mean as \pm SD. ${ }^{*} p<0.05 ;{ }^{* *} p<0.01 ;{ }^{* *} p<0.001$.

IVSd: Interventicular Septal Diastolic Dimension; IVSs: Interventricular Septal Systolic Dimension; LAVI: Left Atrial Volume Index; LVEF: Left Ventricular Ejection Fraction; PASP: Pulmonary Artery Systolic Pressure; TAPSE: Tricuspid Annular Plane Systolic Excursion.

Table 5: Correlation between total serum protein (TSP) and echocardiographic characteristics.

\begin{tabular}{|l|c|c|}
\hline \multicolumn{1}{|c|}{ Parameter } & $\begin{array}{c}\text { TSP in Obese/Ow } \\
\text { Group }(\mathbf{N}=64)\end{array}$ & $\begin{array}{c}\text { TSP in Control } \\
\text { Group }(\mathbf{N}=\mathbf{2 4})\end{array}$ \\
\hline LVEF $(\%)$ & $-0.84^{* *}$ & -0.72 \\
\hline LV diastolic dimension $(\mathrm{mm})$ & $-0.61^{*}$ & -0.58 \\
\hline Relative wall thickness & $0.57^{*}$ & 0.53 \\
\hline LV mass index $\left(\mathrm{g} / \mathrm{m}^{2}\right)$ & $0.63^{*}$ & 0.59 \\
\hline E/A ratio & $-0.57^{*}$ & -0.54 \\
\hline LAVI $\left(\mathrm{ml} / \mathrm{m}^{2}\right)$ & $0.65^{* *}$ & 0.63 \\
\hline PASP $(\mathrm{mmHg})$ & $0.71^{* *}$ & 0.66 \\
\hline IVSs $(\mathrm{mm})$ & $0.54^{*}$ & 0.5 \\
\hline IVSd $(\mathrm{mm})$ & $0.58^{*}$ & 0.59 \\
\hline
\end{tabular}

${ }^{*} \mathrm{p}<0.05 ;{ }^{* *} \mathrm{p}<0.001$.

IVSd: Interventicular Septal Diastolic Dimension; IVSs: Interventricular Septal Systolic Dimension; LAVI: Left Atrial Volume Index; LVEF: Left Ventricular Ejection Fraction; PASP: Pulmonary Artery Systolic Pressure; TAPSE: Tricuspid Annular Plane Systolic Excursion.

Table 6: Multiple regression analysis of some echocardiographic parameters (pooled groups).

\begin{tabular}{|l|c|c|c|}
\hline \multirow{2}{*}{ Parameter } & \multicolumn{3}{c|}{ TSP } \\
\cline { 2 - 4 } & $\mathbf{r}$ & tc & ß coefficient \\
\hline LVEF $(\%)$ & $-0.39^{*}$ & 0.41 & 0.37 \\
\hline LV diastolic dimension $(\mathrm{mm})$ & $-0.48^{* *}$ & 0.59 & 0.22 \\
\hline Relative wall thickness & $0.52^{* *}$ & 0.24 & 0.19 \\
\hline LV mass index $\left(\mathrm{g} / \mathrm{m}^{2}\right)$ & $0.49^{* *}$ & 0.37 & 0.08 \\
\hline E/A ratio & $-0.38^{* *}$ & 0.31 & 0.17 \\
\hline LAVI $\left(\mathrm{ml} / \mathrm{m}^{2}\right)$ & $0.52^{*}$ & 0.43 & 0.16 \\
\hline PASP $(\mathrm{mmHg})$ & $0.49^{* *}$ & 0.95 & 0.25 \\
\hline IVSs $(\mathrm{mm})$ & $0.37^{*}$ & 0.44 & 0.19 \\
\hline IVSd $(\mathrm{mm})$ & $0.51^{*}$ & 0.13 & 0.05 \\
\hline
\end{tabular}

${ }^{*} \mathrm{p}<0.05 ;{ }^{* *} \mathrm{p}<0.001$.

IVSd: Interventicular Septal Diastolic Dimension; IVSs: Interventricular Septal Systolic Dimension; LAVI: Left Atrial Volume Index; LVEF: Left Ventricular Ejection Fraction; PASP: Pulmonary Artery Systolic Pressure; r: Correlation Coefficient; TAPSE: Tricuspid Annular Plane Systolic Excursion; tc: Thigh Circumference; TSP: Total Serum Protein. 
Table 7: Correlation of TSP and AGR with chosen metabolic and echocardiographic parameters.

\begin{tabular}{|c|c|c|c|c|}
\hline \multirow{2}{*}{ Parameter } & $\begin{array}{l}\text { TSP in Obese/Ow Group } \\
(n=64)\end{array}$ & $\begin{array}{l}\text { TSP in Control Group } \\
(n=24)\end{array}$ & $\begin{array}{l}\text { AGR in Obese/Ow Group } \\
(n=64)\end{array}$ & $\begin{array}{l}\text { AGR in Control Group } \\
(n=24)\end{array}$ \\
\hline & $r$ & $r$ & $\mathbf{r}$ & $\mathbf{r}$ \\
\hline Total cholesterol (mmol/l) & $0.47^{\star}$ & 0.42 & $0.25^{\star \star}$ & 0.21 \\
\hline Albumin (g/l) & $0.68^{\star}$ & 0.59 & $0.57^{*}$ & 0.55 \\
\hline LVEF (\%) & $-0.84^{\star \star}$ & -0.72 & $-0.10^{\star}$ & -0.1 \\
\hline LV diastolic dimension (mm) & $-0.61^{*}$ & -0.58 & $0.52^{\star}$ & 0.47 \\
\hline Relative wall thickness & $0.57^{\star}$ & 0.53 & $0.48^{\star}$ & 0.43 \\
\hline LV mass index $\left(\mathrm{g} / \mathrm{m}^{2}\right)$ & $0.63^{\star}$ & 0.59 & $0.55^{\star}$ & 0.52 \\
\hline E/A ratio & $-0.57^{*}$ & -0.54 & $-0.23^{\star}$ & -0.22 \\
\hline LAVI $\left(\mathrm{ml} / \mathrm{m}^{2}\right)$ & $0.65^{\star \star}$ & 0.63 & $0.58^{\star \star}$ & 0.56 \\
\hline PASP $(\mathrm{mmHg})$ & $0.71^{\star \star}$ & 0.66 & $0.29 *$ & 0.28 \\
\hline IVSs (mm) & $0.54^{\star}$ & 0.5 & $0.13^{*}$ & 0.1 \\
\hline IVSd (mm) & $0.58^{*}$ & 0.59 & $0.11^{*}$ & 0.11 \\
\hline
\end{tabular}

${ }^{*} \mathrm{p}<0.05 ;{ }^{* *} \mathrm{p}<0.001$.

IVSd: Interventicular Septal Diastolic Dimension; IVSs: Interventricular Septal Systolic Dimension; LAVI: Left Atrial Volume Index; LVEF: Left Ventricular Ejection Fraction; PASP: Pulmonary Artery Systolic Pressure; r: Correlation Coefficient; TAPSE: Tricuspid Annular Plane Systolic Excursion; TSP: Total Serum Protein.

Table 8: Correlation of TSP with chosen metabolic and echocardiographic parameters in the individual subgroups with and without HFpEF.

\begin{tabular}{|c|c|c|c|}
\hline \multirow{2}{*}{ Parameter } & $\begin{array}{l}\text { TSP in Obese/Ow Group } \\
\text { With HFpEF }(n=4)\end{array}$ & $\begin{array}{l}\text { TSP in Obese/Ow Group } \\
\text { Without HFpEF }(n=60)\end{array}$ & $\begin{array}{l}\text { TSP in Control Group } \\
\text { Without HFpEF }(n=23)\end{array}$ \\
\hline & $\mathbf{r}$ & $\mathbf{r}$ & $\mathbf{r}$ \\
\hline Total cholesterol (mmol/l) & $0.49^{*}$ & $0.45^{\star}$ & 0.41 \\
\hline Albumin $(g / l)$ & $0.66^{*}$ & $0.63^{*}$ & 0.57 \\
\hline LVEF (\%) & $-0.84^{* *}$ & $-0.82^{* *}$ & -0.7 \\
\hline LV diastolic dimension (mm) & $-0.61^{*}$ & $-0.62^{*}$ & -0.58 \\
\hline Relative wall thickness & $0.58^{*}$ & $0.54^{*}$ & 0.53 \\
\hline LV mass index $\left(\mathrm{g} / \mathrm{m}^{2}\right)$ & $0.67^{*}$ & $0.59^{*}$ & 0.58 \\
\hline E/A ratio & $-0.55^{\star}$ & $-0.55^{\star}$ & -0.51 \\
\hline LAVI $\left(\mathrm{ml} / \mathrm{m}^{2}\right)$ & $0.61^{* *}$ & $0.61^{\star *}$ & 0.66 \\
\hline PASP (mmHg) & $0.73^{\star *}$ & $0.69 * *$ & 0.63 \\
\hline IVSs (mm) & $0.55^{\star}$ & $0.50^{*}$ & 0.51 \\
\hline IVSd (mm) & $0.56^{*}$ & $0.57^{\star}$ & 0.54 \\
\hline
\end{tabular}

${ }^{*} \mathrm{p}<0.05 ;{ }^{* *} \mathrm{p}<0.001$.

IVSd: Interventicular Septal Diastolic Dimension; IVSs: Interventricular Septal Systolic Dimension; LAVI: Left Atrial Volume Index; LVEF: Left Ventricular Ejection Fraction; PASP: Pulmonary Artery Systolic Pressure; r: Correlation Coefficient; TAPSE: Tricuspid Annular Plane Systolic Excursion; TSP: Total Serum Protein.

\section{Discussion}

\section{Main finding}

In the present study, we report for the first time a strong positive correlation between circulating TSP and the occurrence of HFpEF. More importantly, these results are demonstrated among obese pediatric population. In the literature, there are limited data available about the prognostic significance of TSP in the field of cardiovascular disease, not to mention in children. This fact is amplified by low prevalence of HFpEF in children (0.5\%) [11]. our findings also indicated that obesity is may be considered as one of the possible markers of a higher risk of HFpEF development, as similar as TSP, which level was higher in obese children compared to group with normal weight.

Although the study might be limited by follow-up duration, the present data provided novel and important information with regard to the key issue whether the baseline TSP concentration can be a marker for predicting the possible clinical outcomes of obese children's HFpEF as a consequence of the obesity. In addition, these results should provide a basis for better understanding the pathological influence of obesity in HFpEF development in children.

\section{Possible mechanisms}

First of all, obesity is characterized by the accumulation of adipose tissue, associated with chronic low-grade inflammatory process [60]. It has already been demonstrated that most of the inflammatory obesity-related proteins are directly produced by the adipose tissue [61]. The question raises whether the increased serum levels of TSP in obesity depend on an altered metabolic status affecting TSP release by the liver or on a direct production from the adipose tissue [62]. Here, we speculate that TSP could constitute an important link between obesity and its comorbidities (HFpEF) by mediating some of the inflammatory effects associated with obesity status. Recently, the contribution of chronic inflammation to HFpEF has been described [63]. 
Previously, the correlation between sAlb and sGlb with HF has been emphasised. sAlb and sGlb, the two major components of serum proteins, have been confirmed to be involved in the systemic inflammatory process. sAlb indicates nutritional status and relates to chronic inflammation in HF $[64,65]$. Moreover, increased levels of sGlb could serve as a marker of chronic inflammation response and reflect a cumulative exposure of various proinflammatory cytokines [46]. sAlb is a negative-phase reactant and its synthesis is decreased in both acute and chronic inflammation. Chronic inflammation is also known because acute-phase proteins increase. It is a critical contributor to HF occurrence, development and survival, and is also related to the risk of recurrence among patients with HF [66]. Thus, TSP considered as a marker of immune status may be also a marker of HF risk.

Another alternative explanation is that comorbidities associated with development of HF are also associated with worsening TSP profile. These observations suggest its interesting role as a strong surrogate marker for incident HF among the children, a marker that possibly integrates known and unexplored pathways. Regan et al. investigated more than 500 different serum proteins in patients with HFpEF [67]. They have found that biomarkers of angiogenesis, fibrosis, fatty acid metabolism and inflammation are associated with HFpEF and improve discriminative capabilities on top of clinical factors and NT-proBNP. These findings highlight the importance of these pathways in HFpEF and identify potential novel circulating diagnostic biomarkers. Further insight may impact future therapeutic interventions.

In general, the mechanisms underlying TSP changes in obesity, HF and other diseases are not clearly understood. There are many pathological conditions that may influence it. Increase in TSP is associated with dehydration (sAlb likely to be also elevated, too), chronic infection or inflammation (e.g. AIDS, hepatitis, osteomyelitis, endocarditis), paraproteinemia (e.g. myeloma and other causes) or autoimmune disorders (e.g. rheumatoid disease, systemic lupus erythematosus) except 'organ specific' autoimmune diseases without

Autoimmune hepatitis. Low levels only occur as a result of conditions causing low values of the major components, i.e. albumin and the immunoglobulins - particularly IgG (e.g. glomerulonephritis). A low TSP but normal albumin may be the first indication that a patient has humoral immunodeficiency [68].

What is more, Castleberry et al. found that obesity itself was not a risk factor for the pediatric cardiomyopathy population as a whole, including symptomatic and asymptomatic individuals [69]. High circulating lipoprotein levels in obese patients may bind and detoxify lipopolysaccharides that play a role in stimulating the release of inflammatory cytokines, all of which may serve to protect them [70,71].

TSP is commonly measured (1) to diagnose nutrition-related chronic deficiencies [72], (2) as a liver function test to support the diagnosis of liver or kidneys disorders which can stimulate the increase or inhibition of its production (meanwhile, the TSP levels are usually decreased in conditions that are commonly associated with liver or kidneys dysfunction), (3) to diagnose chronic inflammations or infections, haematological and (auto) immunodeficiency disorders [73]. However, though TSP has been considered as a biomarker in many other aspects, its role in human cardiovascular physiology remains unknown in the pediatric population nor adults compared to its individual fractions.

Overweight and obese children and adults have elevated serum levels of C-reactive protein, interleukin-6, tumor necrosis factor- $\alpha$, and leptin [74]. In addition, the concentrations of fibrinogen, orosomucoid, alpha1-antitrypsin, haptoglobin, ceruloplasmin, alpha 1 -acid glycoprotein, growth hormone binding protein, ferritin and retinol-binding protein 4 , increase with increasing weight [75-77]. It has also been demonstrated that obesity is associated with an increased TSP profile, suggesting the role of body's regulatory mechanism to maintain the adequate protein levels in the serum $[78,79]$.

It has been shown that AGR, the most frequently used biomarker from this group, is associated with subsequent stroke, myocardial infarction or vascular death $[80,81]$. On the other hand, the relation between AGR and survival has not been well described in patients with chronic HF. At least, Verma et al. noted the relation between AGR and outcomes in HF patients - low AGR was associated with high 6- and 12-month mortality [82]. Moreover, patients with low AGR were at higher risk of readmission due to HF [83].

Previous studies have also demonstrated that hypoalbuminemia was associated with impaired survival in patients with HF [84-92]. However, no study investigated the cumulative effect of TSP on patients with HF nor HFpEF. It is in agreement with our findings as we described below.

It needs to be emphasized that all mentioned studies were realized with adult patients and not with children. It shows a huge potential for further investigations. In conclusion, our research presents a first step in this extensive sphere.

\section{Clinical implications}

Data reported here allow us to add TSP to the growing list of circulating protein raised in human obesity and somehow involved not only in inflammation and/or in immune response (TNF-, IL6, CRP, and leptin), but also in HFpEF (CRP, NT-proBNP, NTproANP, neuropilin, osteopontin) [93-96].

The results of the present study indicate that TSP may provide a clinically useful tool in combination with other standardized clinical, laboratory and imaging predictors of HFpEF in obese children. The prediction of HFpEF risk is a cornerstone of its future management - accurately provided risk evaluation can be of benefit to patients. Patients with worse prognosis might prosper more from an aggressive treatment and a closer follow-up [97]. There exist previous risk models for patients with HF, which adopted a systems biology approach $[98,99]$. Incorporating information from demographic, biomarker, genomic, proteomic and the initial response to therapy might create a more effective prediction model and hopefully aid in understanding HFpEF prognosis. Hence,

Designing a simple survival model based on routine blood biochemical indexes for clinicians is helpful for better identification of patients at high HFpEF risk.

As a clinical prognostic factor, simplicity is indispensable for daily use. Thus, TSP is a more easily available biomarker which possibly provides a more-accurate HFpEF risk evaluation of obese pediatric 
populational group than other objective indices, possibly allowing early implementation of appropriate intervention in daily practice and leads to better outcomes in patients with higher HF risk.

Further validation of the diagnostic accuracy of this approach will require extensive testing in greater numbers of patients at multiple locations as well as a prognostic cohort. It is possible that inclusion of TSP with other predictors of HFpEF will enhance both the fidelity and the efficacy of this approach for diagnostic purposes.

\section{Our recommendations}

HFpEF remains challenging to diagnose despite advances in cardiac biomarkers, non-invasive imaging modalities, and provocative testing. Fundamentally, it is important to recognize that part of the problem is that HFpEF is a clinical syndrome with a multitude of contributing risk factors, causes, and phenotypic manifestations. Hence, we propose the following focus areas for future research: (1) determination of TSP for HFpEF progression and clinical trajectories, (2) the potential role of TSP to distinguish HFpEF from non-cardiac causes of dyspnea or other comorbidities, (3) prospective validation of TSP as a potential component of some proposed diagnostic algorithm. Of these focus areas, we would like to highlight that something as fundamental as the relationship between chosen biomarker (including TSP) clinical evaluation and prognosis of HFpEF in pediatric (not only obese) population remains largely understudied. How does TSP level relate to different population groups with HFpEF, and are there potential therapeutic implications? In sum, academic institutions with the capacity for advanced diagnostic testing, should prioritize research into development of novel diagnostic tools for HFpEF in (obese) children.

\section{Study Limitations}

Several limitations of this study should be acknowledged. First, we could not assess this causal relationship because of the retrospective study design. Follow-up studies are merited to investigate the TSP levels as a prospective risk factor of obesity and/or HFpEF. Second, there was no detailed information about nutritional or dietary status recorded which may influence TSP concentration, especially state of hydration - meaningful interpretation of results requires that a patient's hydration state is normal. Third, we measured only TSP and AGR from a group of serum proteins. Furthermore, TSP was measured once, on the day of the admission. Assessment of additional measurements, as well as markers including prothrombotic proteins, the fibrinogen, prothrombin, and other inflammatory proteins would improve the reliability of our results, as well as no laboratory screening toward other comorbidities including immune disease, plasma cell tumors or parasitic disease was done. Fourth, detailed echocardiographic assessments were not performed in the present study because of its retrospective design. Advanced echocardiography may help to clarify the relationship not only between TSP and previously mentioned parameters including LVEF, but also with others. Additionally, all examined parameters were derived from adults due to limited data known in the pediatric population. Finally, our analysis was based on a relatively small cohort from one rural area and limited to population from the School of obesity programme. The findings of our study may not be generalizable to the entire population. Despite these limitations, our work represents the first study that focuses on the predictive value of TSP in diagnostic of
HFpEF in obese children.

\section{Conclusions}

In conclusion, our findings suggest that higher TSP levels are positively associated with obesity and HFpEF in children and could be used as a biomarker that not only serve as a screening and/or prediction tool of development of new onset hepatic, renal or nutritional insufficiency, but also to identify individuals with HFpEF. The novel observation of these results may provide a clue to further elucidating the pathophysiology and appropriate diagnostics of HFpEF. We believe that the present study provides the basis for future studies on this disease.

Although our report provides novel and useful clinical informations of pediatric (obese) patients with HFpEF, some important clinical problems remain unsolved. As discussed, the patient's nutritional state may be highly related to the occurrence of HFpEF, as well as the importance of TSP in this problem. A more precise nutritional evaluation could provide the answer to this question. Another important question is whether monitoring of TSP levels may be effective in diagnostics and potential treatment of HFpEF. In other words, the reason for elevation of TSP levels in (obese) pediatric patients with HFpEF is still not precisely known. Furthermore, it is also unclear whether TSP elevation will be reversible when HFpEF spontaneously improves. In order to provide a diagnostic and therapeutic strategy for HFpEF, a physiological or biochemical approach and consideration of these questions are essential. Future prospective studies would provide the answer to these questions.

The clinical recognition and understanding of HFpEF in children have just started. Thus, correct diagnosis and careful observation of (obese) pediatric patients with this condition will provide more precise clinical information about this type of HF.

\section{Acknowledgements}

We thank Alzbeta Banovcinova, MD for her contribution to data collection.

\section{References}

1. Paulus WJ, Tschope C, Sanderson JE, et al. How to diagnose diastolic heart failure: A consensus statement on the diagnosis of heart failure with normal left ventricular ejection fraction by the Heart Failure and Echocardiography Associations of the European Society of Cardiology. Eur Heart J. 2007; 28: 2539-2550.

2. Owan TE, Redfield MM. Epidemiology of diastolic heart failure. Prog Cardiovasc Dis. 2005; 47: 320-332.

3. Owan TE, Hodge DO, Herges RM, Jacobsen SJ, Roger VL, Redfield MM. Trends in prevalence and outcome of heart failure with preserved ejection fraction. N Engl J Med. 2006; 355: 251-259.

4. Kitzman DW, Little WC, Brubaker PH, et al. Pathophysiological characterization of isolated diastolic heart failure in comparison to systolic heart failure. JAMA. 2002; 288: 2144-2150.

5. Zile MR, Baicu CF, Gaasch WH. Diastolic heart failure--abnormalities in active relaxation and passive stiffness of the left ventricle. $\mathrm{N}$ Engl $\mathrm{J}$ Med. 2004; 350: 1953-1959.

6. Kass DA, Bronzwaer JG, Paulus WJ. What mechanisms underlie diastolic dysfunction in heart failure?Circ Res. 2004; 94: 1533-1542.

7. Lam CS, Donal E, Kraigher-Krainer E, Vasan RS. Epidemiology and clinical course of heart failure with preserved ejection fraction. Eur J Heart Fail. 2011; 


\section{3: $18-28$.}

8. Little WC. Heart failure with a normal left ventricular ejection fraction: diastolic heart failure. Trans Am Clin Climatol Assoc. 2008; 119: 93-102.

9. Maurer MS, Burkhoff D, Fried LP, Gottdiener J, King DL, Kitzman DW. Ventricular structure and function in hypertensive participants with heart failure and a normal ejection fraction: the Cardiovascular Health Study. J Am Coll Cardiol. 2007; 49: 972-981.

10. Zile MR, Gaasch WH, Anand IS, et al. Mode of death in patients with heart failure and a preserved ejection fraction: results from the Irbesartan in Hear Failure with Preserved Ejection Fraction Study (I-Preserve) trial. Circulation. 2010; 121: 1393-1405

11. Masutani S, Saiki H, Kurishima C, Ishido H, Tamura M, Senzaki H. Heart failure with preserved ejection fraction in children: hormonal imbalance between aldosterone and brain natriuretic peptide. Circ J. 2013; 77: 2375 2382.

12. World Health Organization. Obesity: Preventing and managing the global epidemic, report of a WHO Consultation. Geneva: World Health Organization 2004.

13. Kenchaiah S, Evans JC, Levy D, et al. Obesity and the risk of heart failure. $\mathrm{N}$ Engl J Med. 2002; 347: 305-313.

14. Obokata M, Reddy YNV, Pislaru SV, et al. Evidence Supporting the Existence of a Distinct Obese Phenotype of Heart Failure With Preserved Ejection Fraction. Circulation. 2017; 136: 6-19.

15. Tsujimoto T, Kajio H. Abdominal Obesity is associated with an increased risk of all-cause mortality in patients with HFpEF. J Am Coll Cardiol. 2017; 70: 2739-2749.

16. Jelic S, Lederer DJ, Adams T, et al. Vascular inflammation in obesity and sleep apnea. Circulation. 2010; 121: 1014-1021.

17. Taube A, Schlich R, Sell H, Eckardt K, Eckel J. Inflammation and metabolic dysfunction: links to cardiovascular diseases. Am J Physiol Heart Circ Physiol. 2012; 302: H2148-H2165.

18. Paulus WJ, Tschöpe C. A novel paradigm for heart failure with preserved ejection fraction: comorbidities drive myocardial dysfunction and remodeling through coronary microvascular endothelial inflammation. J Am Coll Cardiol. 2013; 62: 263-271.

19. Kalogeropoulos A, Georgiopoulou V, Psaty BM, et al. Inflammatory markers and incident heart failure risk in older adults: the Health ABC (Health, Aging, and Body Composition) study. J Am Coll Cardiol. 2010; 55: 2129-2137.

20. Ho JE, Lyass A, Lee DS, et al. Predictors of new-onset heart failure: differences in preserved versus reduced ejection fraction. Circ Heart Fail. 2013; 6: 279-286.

21. Brouwers FP, de Boer RA, van der Harst $P$, et al. Incidence and epidemiology of new onset heart failure with preserved vs. reduced ejection fraction in a community-based cohort: 11-year follow-up of PREVEND. Eur Heart J. 2013; 34: $1424-1431$

22. Gupta DK, Shah AM, Castagno D, et al. Heart failure with preserved ejection fraction in African Americans: The ARIC (Atherosclerosis Risk in Communities) study. JACC Heart Fail. 2013; 1: 156-163.

23. Wang YC, Liang CS, Gopal DM, et al. Preclinical Systolic and Diastolic Dysfunctions in Metabolically Healthy and Unhealthy Obese Individuals. Circ Heart Fail. 2015; 8: 897-904.

24. Russo C, Jin Z, Homma S, et al. Effect of obesity and overweight on left ventricular diastolic function: a community-based study in an elderly cohort. J Am Coll Cardiol. 2011; 57: 1368-1374.

25. Lavie CJ, Alpert MA, Arena R, et al. Impact of obesity and the obesity paradox on prevalence and prognosis in heart failure. JACC Heart Fail. 2013; 1: 93102.

26. Oreopoulos A, Padwal R, Kalantar-Zadeh K, et al. Body mass index and mortality in heart failure: a meta- analysis. Am Heart J. 2008; 156: 13-22.

27. Horwich TB, Fonarow GC, Hamilton MA, et al. The relationship between obesity and mortality in patients with heart failure. J Am Coll Cardiol. 2001; 38: 789-795.

28. Lissin LW, Gauri AJ, Froelicher VF, et al. The prognostic value of body mass index and standard exercise testing in male veterans with congestive heart failure. J Card Fail. 2002; 8: 206-215.

29. Haass M, Kitzman DW, Anand IS, et al. Body mass index and adverse cardiovascular outcomes in heart failure patients with preserved ejection fraction: results from the Irbesartan in Heart Failure with Preserved Ejection Fraction (I-PRESERVE) trial. Circ Heart Fail. 2011; 4: 324-331.

30. Powell-Wiley TM, Ngwa J, Kebede S, et al. Impact of body mass index on heart failure by race/ethnicity from the get with The Guidelines-Heart Failure (GWTG-HF) Registry. JACC Heart Fail. 2018; 6: 233-242.

31. Padwal R, McAlister FA, McMurray JJ, et al. The obesity paradox in heart failure patients with preserved versus reduced ejection fraction: a metaanalysis of individual patient data. Int J Obes. 2014; 38: 1110-1114.

32. Adamopoulos C, Meyer P, Desai RV, et al. Absence of obesity paradox in patients with chronic heart failure and diabetes mellitus: a propensitymatched study. Eur J Heart Fail. 2011; 13: 200-206.

33. Pozzo J, Fournier $\mathrm{P}$, Lairez $\mathrm{O}$, et al. Obesity paradox: origin and best way to assess severity in patients with systolic HF. Obesity. 2015; 23: 2002-2008.

34. Vest AR, Wu Y, Hachamovitch R, et al. The heart failure overweight/obesity survival paradox: the missing sex link. JACC Heart Fail. 2015; 3: 917-926.

35. Horwich TB, Fonarow GC, Clark AL. Obesity and the Obesity Paradox in Heart Failure. Prog Cardiovasc Dis. 2018; 61: 151-156.

36. Nagarajan V, Kohan L, Holland E, et al. Obesity paradox in heart failure: a heavy matter. ESC Heart Fail. 2016; 3: 227-234.

37. Carbone S, Lavie CJ, Arena R. Obesity and heart failure: focus on the obesity paradox. Mayo Clin Proc. 2017; 92: 266-279.

38. Tadic M, Cuspidi C. Obesity and heart failure with preserved ejection fraction: a paradox or something else? Heart Fail Rev. 2019; 24: 379-385.

39. Wang TJ. The obesity paradox in heart failure: weighing the evidence. J Am Coll Cardiol. 2014; 64: 2750-2752.

40. Lupón J, de Antonio M, Vila J, et al. Development of a novel heart failure risk tool: the barcelona bio-heart failure risk calculator (BCN bio-HF calculator). PLoS One. 2014; 9: e85466.

41. Levy WC, Mozaffarian D, Linker DT, et al. The Seattle Heart Failure Model: prediction of survival in heart failure. Circulation. 2006; 113: 1424-1433.

42. Mozaffarian D, Anker SD, Anand I, et al. Prediction of mode of death in heart failure: the Seattle Heart Failure Model. Circulation 2007; 116: 392-398.

43. Yancy CW, Jessup M, Bozkurt B, et al. 2013 ACCF/AHA guideline for the management of heart failure: a report of the American College of Cardiology Foundation/American Heart Association Task Force on Practice Guidelines. Circulation. 2013; 128: e240-e327.

44. Lindenfeld J, Albert NM, Boehmer JP, et al. Heart Failure Society of America HFSA 2010 Comprehensive Heart Failure Practice Guideline. J Card Fail. 2010; 16: e1-e194.

45. Ponikowski P, Voors AA, Anker SD, et al. ESC Scien- tific Document Group. 2016 ESC Guidelines for the diagnosis and treatment of acute and chronic heart failure: the task force for the diagnosis and treatment of acute and chronic heart failure of the European Society of Cardiology (ESC) developed with the special contribution of the Heart Failure Association (HFA) of the ESC. Eur Heart J. 2016; 37: 2129-2200.

46. Gabay C, Kushner I. Acute-Phase Proteins and Other Systemic Responses to Inflammation. N Engl J Med Overseas Ed. 1999; 340: 448-454.

47. McPherson, Richard "Chapter 19: Specific proteins" McPherson and Pincus: Henry's clinical diagnosis and management by laboratory methods, 21st Ed. 2006; 262-270.

48. Pieske B, Tschöpe C, de Boer RA, et al. How to diagnose heart failure with preserved ejection fraction: the HFA-PEFF diagnostic algorithm: a consensus 
recommendation from the Heart Failure Association (HFA) of the European Society of Cardiology (ESC). Eur J Heart Fail. 2020; 22: 391-412.

49. World Health Organization. WHO Child Growth Standards: length/heightfor-age, weight-for-age, weight-for-length, weight-for height and body mass index-for-age: methods and development. WHO.2006; 3-11.

50. World Health Organization. Waist circumference and waist-hip ratio: report of a WHO expert consultation, Geneva, 8-11 December 2008. WHO. 2011; 5-7.

51. US Preventive Services Task Force, Grossman DC, Bibbins-Domingo K, et al. Screening for Obesity in Children and Adolescents: US Preventive Services Task Force Recommendation Statement. JAMA. 2017; 317: 2417-2426.

52. Riley M, Hernandez AK, Kuznia AL. High Blood Pressure in Children and Adolescents. Am Fam Physician. 2018; 98: 486-494.

53. Samuels J, Samuel J. New guidelines for hypertension in children and adolescents. J Clin Hypertens (Greenwich). 2018; 20: 837-839.

54. Petersmann A, Müller-Wieland D, Müller UA, et al. Definition, Classification and Diagnosis of Diabetes Mellitus. Exp Clin Endocrinol Diabetes. 2019; 127: S1-S7.

55. Diabetes Canada Clinical Practice Guidelines Expert Committee, Mancini GBJ, Hegele RA, Leiter LA. Dyslipidemia. Can J Diabetes. 2018; 42: S178-S185.

56. Expert Panel on Integrated Guidelines for Cardiovascular Health and Risk Reduction in Children and Adolescents; National Heart, Lung, and Blood Institute. Expert panel on integrated guidelines for cardiovascular health and risk reduction in children and adolescents: summary report. Pediatrics. 2011 128: S213-S256.

57. Zimmet $P$, Alberti KG, Kaufman F, et al. The metabolic syndrome in children and adolescents-an IDF consensus report. Pediatr Diabetes. 2007; 8: 299306.

58. Lopez L, Colan SD, Frommelt PC, et al. Recommendations for quantification methods during the performance of a pediatric echocardiogram: a report from the Pediatric Measurements Writing Group of the American Society of Echocardiography Pediatric and Congenital Heart Disease Council. J Am Soc Echocardiogr. 2010; 23: 465-577.

59. Obokata M, Reddy YNV, Borlaug BA. Diastolic Dysfunction and Heart Failure with Preserved Ejection Fraction: Understanding Mechanisms by Using Noninvasive Methods. JACC Cardiovasc Imaging. 2020; 13: 245-257.

60. Wu H, Ballantyne CM. Metabolic Inflammation and Insulin Resistance in Obesity. Circ Res. 2020; 126: 1549-1564.

61. Trayhurn P, Beattie JH. Physiological role of adipose tissue: white adipose tissue as an endocrine and secretory organ. Proc Nutr Soc. 2001; 60: 329339.

62. Chiellini C, Bertacca A, Novelli SE, et al. Obesity modulates the expression of haptoglobin in the white adipose tissue via TNFalpha. J Cell Physiol. 2002 190: $251-258$.

63. Sanders-van Wijk S, Tromp J, Beussink-Nelson L, et al. Proteomic Evaluation of the Comorbidity-Inflammation Paradigm in Heart Failure With Preserved Ejection Fraction: Results From the PROMIS-HFpEF Study. Circulation. 2020; 142: 2029-2044.

64. Gopal DM, Kalogeropoulos AP, Georgiopoulou VV, et al. Serum albumin concentration and heart failure risk The Health, Aging, and Body Composition Study. Am Heart J. 2010; 160: 279-285.

65. Horwich TB, Kalantar-Zadeh K, MacLellan RW, et al. Albumin levels predic survival in patients with systolic heart failure. Am Heart J. 2008; 155: 883-889.

66. Dick SA, Epelman S. Chronic Heart Failure and Inflammation: What Do We Really Know? Circ Res. 2016; 119: 159-176.

67. Regan J, Kwee L, Grass E, et al. Circulating Protein Biomarkers Associated with Heart Failure with Preserved Ejection Fraction. J Card Fail. 2020; 26 : S2.

68. Kitzman DW, Shah SJ. The HFpEF Obesity Phenotype: The Elephant in the Room. J Am Coll Cardiol. 2016; 68: 200-203.

69. Castleberry CD, Jefferies JL, Shi L, et al. No Obesity Paradox in Pediatric
Patients with Dilated Cardiomyopathy. JACC Heart Fail. 2018; 6: 222-230.

70. Lavie CJ, Mehra MR, Milani RV. Obesity and heart failure prognosis: paradox or reverse epidemiology? Eur Heart J. 2005; 26: 5-7.

71. Rauchhaus M, Coats AJ, Anker SD. The endotoxin lipoprotein hypothesis. Lancet. 2000; 356: 930-933.

72. Tian CR, Qian L, Shen XZ, Li JJ, Wen JT. Distribution of serum total protein in elderly Chinese. PLoS One. 2014; 9: e101242.

73. Katayev A, Balciza C, Seccombe DW. Establishing reference intervals for clinical laboratory test results: is there a better way? Am J Clin Pathol. 2010 133: 180-186.

74. Lakka HM, Lakka TA, Tuomilehto J, Salonen JT. Abdominal obesity is associated with increased risk of acute coronary events in men. Eur Heart J. 2002; 23: 706-713.

75. Engström G, Hedblad B, Stavenow L, Lind P, Janzon L, Lindgärde F. Inflammation-sensitive plasma proteins are associated with future weight gain. Diabetes. 2003; 52: 2097-2101.

76. Pongpaew P, Schelp F, Supawan V, et al. Influence of dietary intake on alpha-2-macroglobulin and other biochemical parameters in healthy thai males. Nutrition Research. 1991; 11: 559-565.

77. Janke J, Engeli S, Boschmann M, et al. Retinol-binding protein 4 in human obesity. Diabetes. 2006; 55: 2805-2810.

78. Riaz M, Alam SS, Raza M, et al. Obesity as risk factor and study of obesity related proteins in diabetes mellitus. Afr J Biotechnol. 2009; 8: 737-744.

79. Madhuvanthi M, Lathadevi GV. Serum Proteins Alteration in Association with Body Mass Index in Human Volunteers. J Clin Diagn Res. 2016; 10: CC05CC7.

80. Azab B, Bibawy J, Harris K, et al. Value of albumin-globulin ratio as a predictor of all-cause mortality after non-ST elevation myocardial infarction. Angiology. 2013; 64: 137-145.

81. Beamer N, Coull BM, Sexton G, de Garmo P, Knox R, Seaman G. Fibrinogen and the albumin-globulin ratio in recurrent stroke. Stroke. 1993; 24: 11331139.

82. Verma B, Williams B, Honushefsky A, Kaur S, Doddamani S. The Albumin Globulin Ratio in Chronic Heart Failure-Implications for Mortality. J Card Fail. 2016; 22: S30

83. Maeda D, Sakane K, Somiya K et al. Albumin-to-Globulin Ratio (AGR) Is Independently Associated with the Heart Failure Readmission. J Card Fail. 2017; 23: S30.

84. Grodin JL, Lala A, Stevens SR, et al. Clinical Implications of Serum Albumin Levels in Acute Heart Failure: Insights From DOSE-AHF and ROSE-AHF. J Card Fail. 2016; 22: 884-890.

85. Nelson JJ, Liao D, Sharrett AR, et al. Serum albumin level as a predictor of incident coronary heart disease: the atherosclerosis Risk in Communities (ARIC) study. Am J Epidemiol. 2000; 151: 468-477.

86. Danesh J, Collins R, Appleby P, Peto R. Association of fibrinogen, C-reactive protein, albumin, or leukocyte count with coronary heart disease: metaanalyses of prospective studies. JAMA. 1998; 279: 1477-1482.

87. Weijenberg MP, Feskens EJ, Souverijn JH, Kromhout D. Serum albumin, coronary heart disease risk, and mortality in an elderly cohort. Epidemiology. 1997; 8: 87-92.

88. Gillum RF, Makuc DM. Serum albumin, coronary heart disease, and death. Am Heart J. 1992; 123: 507-513.

89. Corti MC, Salive ME, Guralnik JM. Serum albumin and physical function as predictors of coronary heart disease mortality and incidence in older persons. J Clin Epidemiol. 1996; 49: 519-526.

90. Kuller LH, Eichner JE, Orchard TJ, Grandits GA, McCallum L, Tracy RP. The relation between serum albumin levels and risk of coronary heart disease in the multiple risk factor intervention trial. Am J Epidemiol. 1991; 134: 12661277. 
91. Djousse' L, Rothman KJ, Cupples LA, Levy D, Ellison RC. Serum albumin and risk of myocardial infarction and all-cause mortality in the Framingham offspring study. Circulation. 2002; 106: 2919-2924.

92. Phillips A, Shaper AG, Whincup PH. Association between serum albumin and mortality from cardiovascular disease, cancer, and other causes. Lancet. 1989; 2: 1434-1436.

93. Sanders-van Wijk S, van Empel V, Davarzani N, et al. Circulating biomarkers of distinct pathophysiological pathways in heart failure with preserved vs. reduced left ventricular ejection fraction. Eur J Heart Fail. 2015; 17: 10061014.

94. van Veldhuisen DJ, Linssen GC, Jaarsma T, et al. B-type natriuretic peptide and prognosis in heart failure patients with preserved and reduced ejection fraction. J Am Coll Cardiol. 2013; 61:1498-1506.

95. Maeder MT, Rickenbacher $\mathrm{P}$, Rickli $\mathrm{H}$, et al. $\mathrm{N}$-terminal pro brain natriuretic peptide-guided management in patients with heart failure and preserved ejection fraction: findings from the Trial of Intensified versus standard medical therapy in elderly patients with congestive heart failure (TIME-CHF). Eur J Heart Fail. 2013; 15: 1148-1156.

96. Tromp J, Khan MA, Klip IT, et al. Biomarker Profiles in Heart Failure Patients With Preserved and Reduced Ejection Fraction. J Am Heart Assoc. 2017; 6: e003989.

97. Ouwerkerk W, Voors AA, Zwinderman AH. Factors influencing the predictive power of models for predicting mortality and/or heart failure hospitalization in patients with heart failure. JACC Heart Fail 2014; 2: 429-436.

98. Giamouzis G, Kalogeropoulos A, Georgiopoulou V, et al. Hospitalization epidemic in patients with heart failure: risk factors, risk prediction, knowledge gaps, and future directions. J Card Fail. 2011; 17: 54-75.

99. Betihavas V, Davidson PM, Newton PJ, et al. What are the factors in risk prediction models for rehospitalization for adults with chronic heart failure? Aust Crit Care 2012; 25: 31-40. 